\title{
INFLUENNCIAS DO CICLO MENSTRUAL NA PERFORMANCE DE ATLETAS: REVISÃO DE LITERATURA
}

\begin{abstract}
Clémersom Neis ${ }^{1}$
Juliana Pizzi ${ }^{2}$

NEIS, C.; PIZZI, J. Influências do ciclo menstrual na performance de atletas: revisão de literatura. Arq. Cienc. Saúde UNIPAR, Umuarama, v. 22, n. 2, p. 123-128, maio/ago. 2018.

RESUMO: A participação das mulheres nos esportes de alto rendimento vem crescendo, porém a literatura ainda é escassa em relação à influência dos aspectos hormonais e o ciclo menstrual (CM) na performance física. Dessa forma o objetivo do presente trabalho foi identificar a influência do CM na performance de atletas por meio de uma pesquisa de revisão da literatura, utilizando duas bases de dados (Biblioteca Virtual em Saúde-Bireme e Scientific Electronic Library Online-Scielo) e as seguintes palavras-chave (Descritores em Ciências da Saúde-DECS): "ciclo menstrual", "ciclo ovariano", "ciclo endometrial”, "atletas", na língua portuguesa e disponível para leitura na íntegra entre e setembro de 2016. Foram encontrados seis artigos após a busca. Em relação aos artigos que relataram a fase específica do CM, quatro artigos avaliaram a correlação de Síndrome Pré-Menstrual (SPM) com o desempenho em atletas durante a fase pré-menstrual, um artigo estudou a variação hormonal nas diferentes fases do CM e outro artigo especificou as disfunções menstruais (oligomenorreia e amenorreia) relacionadas ao treinamento. Constatou-se que o CM interfere no sistema hormonal e em sintomas físicos e emocionais relatados pelas atletas, principalmente na fase pré-menstrual, podendo causar a SPM, e com impacto no desempenho físico.
\end{abstract}

PALAVRAS-CHAVE: Atletas. Ciclo menstrual. Hormônios. Performance.

\section{MENSTRUAL CYCLE INFLUENCES ON ATHLETE PERFORMANCE: A SYSTEMATIC REVIEW}

\begin{abstract}
The participation of women in high performance sports is growing; however, literature is still scarce regarding the influence of hormonal aspects and the menstrual cycle (MC) on physical performance. Identify the influence of MC on the performance of athletes. Literature review using two databases (Virtual Health Library - Medicine ${ }^{\circledR}$ and Scientific Electronic Library Online - Scielo) and the following keywords (Health-DECS Sciences): "menstrual cycle", "ovarian cycle", "endometrial cycle", "athletes", in Portuguese and in full-text available between March and September, 2016. A total of six papers were included after search and screening. Regarding the papers reporting the specific MC phase, four articles evaluated the correlation of Premenstrual Syndrome (PMS) with the performance of athletes during the pre-menstrual phase, one paper studied the hormonal variations in the different MC phases, and another paper specified menstrual dysfunction (oligomenorrhea and amenorrhea) related to training. It could be concluded that MC interferes with the performance of athletes in both the hormonal system and in physical and emotional symptoms reported by the athletes, mainly during the pre-menstrual phase, possibly causing PMS and with an impact on the physical performance.
\end{abstract}

KEYWORDS: Athletes. Hormones. Menstrual cycle. Performance.

\section{Introdução}

A comparação do desempenho entre homem e mulher nas atividades esportivas é sempre um assunto recorrente, visto que a participação das mulheres no esporte tem aumentado numericamente nas últimas décadas. Existem grandes diferenças fisiológicas entre homens e mulheres, como menores valores de força muscular de membros inferiores e superiores nas mulheres (GLASS; STANTON, 2004) e diferentes concentrações plasmáticas dos principais hormônios anabólicos (testosterona, GH e IGF-1), em repouso ou após esforço intenso entre os sexos (BORST et al., 2001). Um estudo indicou que homens e mulheres apresentam comportamentos relativamente diferentes em séries múltiplas de exercício com pesos, com as mulheres apresentando desempenho mais estável e maior capacidade de resistência à fadiga e os mecanismos responsáveis por esse fenômeno, bem como pela queda significativa de desempenho quando homens e mulheres são submetidos à séries múltiplas de exercícios com pesos a uma intensidade constante ( $80 \%$ de $1-\mathrm{RM})$, ainda merecem ser melhor investigados (SALVADOR et al., 2005).

Segundo Barros e Angeli (2012), homens possuem maior quantidade de glóbulos vermelhos no sangue, o que proporciona uma maior capacidade de transporte de oxigênio e consequentemente um desempenho aeróbico e desempenho cardíaco (maior volume de sangue que o coração consegue bombear por minuto) superior ao de mulheres. Estas diferenças proporcionam aos homens uma vantagem fisiológica em qualquer solicitação de esportes de resistência. A literatura científica ainda é escassa sobre os aspectos hormonais que afetam o desempenho de mulheres praticantes de exercício físico (DAVID et al., 2009), pois as alterações hormonais durante o período menstrual podem influenciar na performance dependendo do tipo de atividade que é praticada (PORTAL DA EDUCAÇÃO FÍSICA, 2012).

Para alcançar um patamar ideal de aptidão física é necessário a elaboração de um programa de treinamento periodizado, baseado em quantidade, distribuição e monitoramento adequados das cargas de treinamento promovendo adaptações precisas em relação ao ganho e melhora de rendimento (FREITAS; MILOSKY; BARA FILHO, 2011) e para o treinador utilizar essas respostas físicas durante a progressão das cargas implementadas e periodização futura (NAKAMURA; MOREIRA; AOKI, 2010), pois um programa de treinamento com cargas inadequadas combinado com períodos de recuperação insuficientes resultará em um declí-

${ }^{1}$ Professor de Educação Física e Técnico de Corrida de Rua. Endereço para correspondência: Rua Octaviano Teixeira dos Santos, nº 140 , Bloco 2, apto 301, Bairro Centro, Francisco Beltrão-PR, CEP: 85.601-030, E-mail: clemersom.neis@hotmail.com

${ }^{2}$ Professora Doutora do Curso de Educação Física da Unipar - Unidade de Francisco Beltrão. Rua Videira, nº 67, Bairro São Cristovão, Francisco Beltrão-PR, CEP: 85.601-363, E-mail: jupizzi@prof.unipar.br 
nio de desempenho, levando à síndrome do supertreinamento, o overtraining (BRURINI; OLIVEIRA; BURINI, 2010).

Com isso, torna-se necessário uma abordagem mais cautelosa quando se trata do gênero feminino. Para Borresen e Lambert (2009), um programa mal elaborado pode causar desequilíbrios hormonais e prejuízo na performance física, pois em um programa de treinamento de qualidade deve haver um contínuo desequilíbrio da homeostase do atleta, mas também é de suma importância a aplicação de períodos suficientes de recuperação para a ocorrência da supercompensação. Contudo, o estresse provocado pelo treinamento pode acarretar efeitos negativos, se as cargas utilizadas não forem condizentes com as capacidades do atleta, podendo até mesmo causar danos à sua saúde. É importante considerar as fases do CM e alterações hormonais no planejamento e aplicação de um treinamento para atleta. Portanto, este artigo teve por objetivo identificar a influência do CM na performance de atletas.

\section{Metodologia}

Para realização desta revisão sistemática de literatura, foram utilizadas as bases de dados Biblioteca Virtual em Saúde-Bireme e Scientific Electronic Library Online-Scielo, utilizando as seguintes palavras-chave, encontradas nos Descritores em Ciências da Saúde (DECS): "ciclo menstrual", "ciclo ovariano", "ciclo endometrial", "atletas". As buscas foram por estudos na língua portuguesa, disponível para leitura na íntegra, sem estabelecer limites para ano de publicação, e ocorreu durante o período de Março a Setembro de 2016.

Após terminada a busca, estes passaram por análise e seleção, excluindo os artigos que se encontravam repetidos nas buscas e nas bases de dados (Fluxograma 1).

Fluxograma 1: Artigos incluídos na revisão.

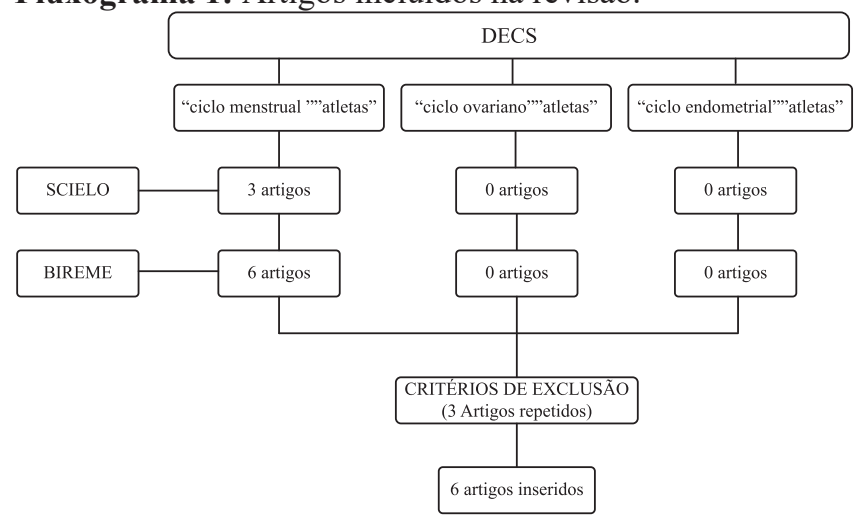

\section{Resultados e Discussão}

Os seis artigos encontrados nesta pesquisa de revisão englobam diversas modalidades esportivas, foram publicados entre 2009 e 2015, com grupos amostrais de sete até 112 atletas e com idade mínima de 12 e máxima de 49 anos. Todos os artigos incluídos nesta pesquisa utilizaram como critério de exclusão atletas usuárias de contraceptivos orais (COs) e todos foram aprovados por Comitês de Ética em Pesquisa com seres humanos.

Com relação a idade da menarca e o início do trei- namento, dois artigos utilizaram como critério de exclusão as atletas que apresentaram menarca há pelo menos um ano (DAVID et al., 2009; VIEIRA; GAION, 2009). Em relação ao tempo de treinamento, quatro artigos especificaram a duração diária e semanal em horas, sendo três deles com doze horas de treinamento semanal (SCHTSCHERBYNA et al., 2012; GAION; VIEIRA, 2010; VIEIRA; GAION, 2009) e um deles com seis horas de treinamento semanal (GAION; VIEIRA, 2010; SILVA, 2009). Todos os artigos incluídos estão descritos na Tabela 1 .

Com relação a influência do CM na performance de atletas, foi encontrado um artigo que analisou detalhadamente as diferentes fases do CM (folicular, ovulatória, lútea) apresentando maior risco de desequilíbrio hormonal e disfunção menstrual em atletas de alto rendimento de Ginástica Rítmica, o que inspira necessidade de atenção clínica (COELHO; SIMÕES; LUNZ, 2015). Outro artigo explicou as disfunções do ciclo regular em atletas nadadoras brasileiras competitivas, concluindo que a presença de disfunções menstruais (oligomenorréia e amenorréia) é mais alta na população de atletas do que na população de não-atletas (SCHTSCHERBYNA et al., 2012), e quatro artigos (GAION; VIEIRA, 2010; DAVID et al., 2009; GAION; VIEIRA; SILVA, 2009; VIEIRA; GAION, 2009) trataram de sintomas emocionais e físicos relacionados a fase pré-menstrual, encontrando resultados semelhantes quando relacionado o impacto que esses sintomas causam no desempenho esportivo.

Os sintomas associados à Síndrome Pré-Menstrual (SPM) mais relatados em quatro artigos (GAION; VIEIRA, 2010; GAION; VIEIRA; SILVA, 2009; DAVID et al., 2009; VIEIRA; GAION, 2009) pelas atletas foram: a ansiedade, irritabilidade, mastalgia e a explosão de raiva.

Quatro artigos avaliaram a correlação da SPM com o desempenho em atletas de diversas modalidades (Futsal, Atletismo, Basquetebol, Ciclismo, Ginástica Rítmica, Handebol, Karatê, Natação, Voleibol, Vôlei de Praia e Xadrez) durante a fase pré-menstrual (GAION; VIEIRA, 2010; GAION; VIEIRA; SILVA, 2009; DAVID et al., 2009; VIEIRA; GAION, 2009). Um artigo estudou a variação hormonal nas diferentes fases do $\mathrm{CM}$ em atletas de alto rendimento de Ginástica Rítmica (COELHO; SIMÕES; LUNZ, 2015). Outro artigo especificou as disfunções menstruais (oligomenorreia e amenorreia) em nadadoras adolescentes competitivas (SCHTSCHERBYNA et al., 2012). 
Tabela 1: Artigos selecionados na busca pelas bases de dados

\begin{tabular}{|c|c|c|c|c|c|c|}
\hline $\mathbf{N}^{\mathbf{0}}$ & Autor/Ano & $\begin{array}{l}\text { Método de } \\
\text { Estudo }\end{array}$ & Amostra/População & $\begin{array}{l}\text { Modalidade } \\
\text { Esportiva }\end{array}$ & Hormônios/Sintomas & Resultados \\
\hline 1 & COELHO et al. 2015 & $\begin{array}{l}\text { Estudo de } \\
\quad \text { caso }\end{array}$ & $\begin{array}{c}\mathrm{N}=7 \text { atletas } \\
\text { Idade média: } 19,4 \pm \\
1,1 \text { anos } \\
\text { Olímpiadas de Beijing } \\
\text { (China), 2008 }\end{array}$ & $\begin{array}{l}\text { Ginástica Rítmica } \\
\text { (GR) de alto } \\
\text { rendimento }\end{array}$ & $\begin{array}{c}\text { Estrogênio; } \\
\text { Progesterona; } \\
\text { Luteinizante; Folículo } \\
\text { estimulante } \\
\text { Testosterona; Oligo/ } \\
\text { Amenorreia }\end{array}$ & $\begin{array}{c}65 \% \text { abaixo do valor } \\
\text { referencial clínico; Próximo } \\
\text { ao limite inferior; Próximo ao } \\
\text { limite inferior; } 10 \% \text { abaixo do } \\
\text { valor referencial; } 30 \% \text { abaixo } \\
\text { do valor referencial; Todas as } \\
\text { atletas relataram }\end{array}$ \\
\hline 2 & $\begin{array}{l}\text { SCHTSCHERBYNA } \\
\text { et al. } 2012\end{array}$ & Transversal & $\begin{array}{c}\mathrm{N}=57 \text { atletas } \\
\text { Idade média: } 14,6 \pm \\
0,2 \text { anos } \\
\text { Campeonatos } \\
\text { Estaduais do Rio de } \\
\text { Janeiro } 2006 \text {-pelo } \\
\text { menos a } 5^{\mathrm{a}} \text { posição } \\
\text { no ranking de suas } \\
\text { categorias etárias }\end{array}$ & $\begin{array}{l}\text { Nadadoras } \\
\text { adolescentes } \\
\text { competitivas } \\
\text { brasileiras }\end{array}$ & $\begin{array}{c}\text { Oligomenorreia } \\
\text { (Menstruação escassa) } \\
\text { Eumenorreia } \\
\text { (Menstruação Normal) }\end{array}$ & $\begin{array}{l}\text { Presença de disfunções } \\
\text { menstruais encontradas em } \\
26,3 \% \text { (15 das } 57 \text { atletas), } \\
\text { encontrou-se relação entre } \\
\text { oligomenorreia e a idade do } \\
\text { início do treinamento }\end{array}$ \\
\hline 3 & GAION et al. 2010 & $\begin{array}{l}\text { Descritivo } \\
\text { do tipo } \\
\text { inquérito }\end{array}$ & $\begin{array}{l}\mathrm{N}=25 \text { atletas - com } \\
\text { presença }(\mathrm{n}=12) \mathrm{e} \\
\text { ausência }(\mathrm{n}=13) \text { de } \\
\text { SPM } \\
\text { Idade entre 18-47 anos } \\
\text { Secretaria de Esportes } \\
\text { de um município do } \\
\text { noroeste do PR, 2007 }\end{array}$ & $\begin{array}{c}\text { Atletismo, } \\
\text { Basquetebol, } \\
\text { Ciclismo, Ginástica } \\
\text { Rítmica, Handebol, } \\
\text { Karatê, Natação, } \\
\text { Voleibol, Vôlei de } \\
\text { Praia e Xadrez }\end{array}$ & $\begin{array}{l}\text { Sintomas emocionais } \\
\text { (depressão, ansiedade, } \\
\text { confusão, irritabilidade, } \\
\text { isolamento e explosão } \\
\text { de raiva) e sintomas } \\
\text { físicos (mastalgia, } \\
\text { desconforto abdominal, } \\
\text { cefaleia e edema) }\end{array}$ & $\begin{array}{c}\mathrm{N}=10 \text { atletas ( } 83 \% \text { SPM) } \\
\text { Quando comparados os grupos } \\
\text { (presença e ausência de SPM), } \\
\text { a mastalgia e o desconforto } \\
\text { abdominal tiveram associação } \\
\text { significativa com presença de } \\
\text { SPM; encontraram associação } \\
\text { significativa entre SPM ( } 83 \% \\
\text { das atletas) e volume de } \\
\text { treinamento semanal }\end{array}$ \\
\hline 4 & VIEIRA et al. 2009 & Transversal & $\begin{array}{l}\mathrm{N}=25 \text { atletas - com } \\
\text { presença }(\mathrm{n}=12) \mathrm{e} \\
\text { ausência }(\mathrm{n}=13) \mathrm{de} \\
\mathrm{SPM} \\
\text { Idade entre } 18-49 \text { anos } \\
\text { Competições estaduais } \\
\text { e nacionais, 2007 }\end{array}$ & $\begin{array}{c}\text { Atletismo, } \\
\text { Basquetebol, } \\
\text { Ciclismo, Ginástica } \\
\text { Rítmica, Handebol, } \\
\text { Karatê, Natação, } \\
\text { Voleibol, Vôlei de } \\
\text { Praia e Xadrez } \\
\end{array}$ & Humor & $\begin{array}{c}\text { Somente atletas com SPM } \\
\text { apresentaram aumento na } \\
\text { alteração total de humor } \\
\text { da última semana para o } \\
\text { último dia, sendo a diferença } \\
\text { significativa no } 1^{\mathrm{a}} \text { ciclo } \\
\text { menstrual }\end{array}$ \\
\hline 5 & DAVID et al. 2009 & Transversal & $\begin{array}{l}\mathrm{N}=31 \text { atletas }- \text { com } \\
\text { presença }(\mathrm{n}=22) \mathrm{e} \\
\text { ausência }(\mathrm{n}=9) \text { de } \\
\mathrm{SPM} \\
\text { Idade entre } 12-18 \text { anos } \\
\text { Estudantes }\end{array}$ & Handebol & $\begin{array}{l}\text { Irritabilidade, } \\
\text { mudança de humor, } \\
\text { cólicas, ansiedade, } \\
\text { choro fácil, tensão } \\
\text { nervosa, compulsão } \\
\text { por alimentos, dores } \\
\text { contínuas, dor de } \\
\text { cabeça, fadiga, } \\
\text { "seios" doloridos, } \\
\text { depressão, insônia, } \\
\text { sensação de perda de } \\
\text { controle, confusão, má } \\
\text { coordenação, inchaços } \\
\text { e outros }\end{array}$ & $\begin{array}{c}\mathrm{N}=22 \text { atletas }(71 \% \mathrm{SPM}) \\
\text { Sintomas mais relatados } \\
\text { foram irritabilidade }(86,4 \%), \\
\text { mudanças de humor }(77,3 \%), \\
\text { cólicas }(72,7 \%) \text { e outros }(59 \%)\end{array}$ \\
\hline 6 & GAION et.al. 2009 & Transversal & $\begin{array}{l}\mathrm{N}=112 \text { atletas }- \text { com } \\
\text { presença }(\mathrm{n}=53) \mathrm{e} \\
\text { ausência }(\mathrm{n}=59) \text { de } \\
\text { SPM } \\
\text { Idade entre 18-31 anos } \\
\text { Taça Brasil de Clubes, } \\
2007\end{array}$ & Futsal & $\begin{array}{c}\text { Sintomas emocionais } \\
\text { (depressão, ansiedade, } \\
\text { confusão, irritabilidade, } \\
\text { isolamento e explosão } \\
\text { de raiva), sintomas } \\
\text { físicos (mastalgia, } \\
\text { desconforto abdominal, } \\
\text { cefaleia e edema) e } \\
\text { outros }\end{array}$ & $\begin{array}{l}\mathrm{N}=53 \text { atletas }(47,32 \% \mathrm{SPM}) \\
\text { Depressão, irritabilidade, } \\
\text { explosão de raiva, mastalgia, } \\
\text { desconforto abdominal, } \\
\text { cefaleia, edema, dificuldade } \\
\text { de concentração e dor } \\
\text { lombar apresentaram riscos } \\
\text { significativos de estarem } \\
\text { diretamente associados à SPM }\end{array}$ \\
\hline
\end{tabular}

O estudo pioneiro que avaliou o efeito da adaptação à diversos agentes (como por exemplo, o exercício físico vigoroso) que podem prejudicar os ovários e seu funcionamento, em ratas realizou procedimento experimental que correlacionou o exercício físico com disfunção menstrual por Selye em 1939 (PARDINI, 2001). A influência do ciclo menstrual no rendimento esportivo parece obedecer um caráter individual, pois algumas atletas podem ter seu rendimento esportivo prejudicado na fase pré-menstrual, em virtude do desconforto de seus sintomas e um melhor rendimento no pe- ríodo pós-menstrual em razão do efeito hormonal (FLECK; KRAEMER, 2014). Nesta pesquisa, quatro artigos (GAION; VIEIRA, 2010; DAVID et al., 2009; GAION; VIEIRA; SILVA, 2009; VIEIRA; GAION, 2009) relataram a dificuldade de manter uma qualidade no treinamento durante o período pré-menstrual e menstrual. Dessa forma, com a aplicação de um monitoramento de treinamento adequado, pode-se obter uma melhoria nos componentes da aptidão física necessárias para uma melhoria no desempenho (NUNES et al., 2011).

As capacidades e habilidades físicas e a influên- 
cia do CM não foram relatadas nesta pesquisa aumentando a lacuna científica de estudos para a população feminina e performance em esportes. Estudos recentes com mulheres não-atletas apontam a não influência do $\mathrm{CM}$ na flexibilidade articular. Chaves et al (2002), Melegario et al (2006), Teixeira et al (2012). Porém, diferenças significativas $(p=0,003)$ foram encontradas quando avaliadas 10 atletas de handebol pelo teste do flexômetro, que faziam uso de COs entre as fases ovulatória e anovulatória, encontrando maior flexibilidade na fase anovulatória (pós-menstrual) concluindo que tais achados podem estar relacionados ao aumento da temperatura corporal basal resultante do aumento de progesterona (BISI et al., 2009). Loureiro et al (2011) e Dias, Simão e Da Silva Novaes (2005) avaliaram mulheres saudáveis que faziam uso de COs em testes de 10 repetições máximas, e constataram que as fases do CM não influenciam o desempenho da força muscular nos exercícios resistidos, sejam eles uni ou multiarticulares, para grandes os pequenos grupamentos musculares ou em diferentes segmentos corporais.

Por convenção o CM regular inicia no primeiro dia de menstruação, e ocorre usualmente a cada 28 dias, podendo ser dividido em três fases distintas: folicular, ovulatória e lútea (CHAVES; SIMÃO; ARAÚJO, 2002), sendo estabelecida a fase folicular $1^{\circ}$ ao $9^{\circ}$ dia, fase ovulatória, $10^{\circ}$ ao $14^{\circ}$ dia e fase lútea $15^{\circ}$ ao $28^{\circ}$ dia (WOJTYS et al., 1998). Na fase folicular e ovulatória, o Estradiol, Luteinizante (LH) e Folículo Estimulante (FSH) atingem seu máximo, enquanto na fase lútea ocorre o predomínio de progesterona e em menor grau do estrogênio (DE JONGE, 2003). Nesta pesquisa, foi encontrado um artigo (SCHTSCHERBYNA et al., 2012) que mencionou os hormônios femininos gonadotróficos e seus valores percentuais após realização de treinamento de atletas de elite em Ginástica Rítmica de nível olímpico, relatando disfunção menstrual e expressiva variação hormonal intra e entre indivíduos.

Existe um consenso de que as oscilações hormonais ocorridas nas mulheres devido à alterações, principalmente do estrogênio e progesterona, durante o CM afetam a fisiologia feminina. Em relação ao exercício físico, esse perfil endócrino pode alterar diversos fatores, tais como a utilização de substrato energético em diferentes intensidades e volumes de treinamento, consumo de oxigênio, armazenamento de substratos energéticos e oxidação de gorduras (BRAUN; HORTON, 2001). Estudos sugerem que a fase do ciclo menstrual pode afetar de maneira importante o desempenho físico de atletas, tanto no desempenho aeróbio quanto na força muscular (DE CARVALHO et al., 2014).

Quando levadas em consideração as alterações hormonais do CM em relação à atividades de endurance um estudo feito por Sanborn, Martin e Wagner (1982) encontrou uma frequência de amenorreia de 12,3\% em nadadoras, $12,1 \% \mathrm{em}$ ciclistas e $25,7 \%$ em corredoras, sendo que as corredoras foram o único grupo que apresentou uma correlação positiva entre distância percorrida por treino semanal e amenorreia. Porém em um estudo mais recente feito por Cesar, Pardini e Barros (2001) avaliou o efeito do exercício de endurance no ciclo menstrual de corredoras e os resultados sugeriram que, apesar das grandes distâncias percorridas, o treinamento em intensidade abaixo do limiar anaeróbio, de todas as corredoras contribuiu para importantes benefícios fisiológicos, sem nenhum prejuízo funcional indicando que esses resultados estariam relacionados ao fato de as atletas terem respeitado a intensidade do limiar anaeróbio no treinamento.

Estudos apontam que a prevalência de amenorreia em não-atletas é de 2 a $5 \%$, enquanto que em atletas pode chegar a 66\% (MANTOANELLI; VITALLE; AMANCIO, 2002). Dos artigos listados nesta pesquisa, dois relataram as disfunções menstruais oligomenorreia e amenorreia, sendo que em um artigo todas as atletas apresentaram uma dessas duas disfunções, e em outro que investigou somente a presença de oligomenorreia, encontrou 26,3\% das atletas em estudo. Algumas das possíveis causas dessas disfunções são: perda de peso, excesso de treino, quantidade insuficiente de gordura corporal, perda de estoques específicos de gordura corporal e dieta inadequada (MANTOANELLI; VITALLE; AMANCIO, 2002).

Os sintomas físicos e emocionais relatados pelas atletas, em diferentes modalidades, foram os seguintes: ansiedade, irritabilidade, depressão, tensão nervosa, mudanças de humor, sensação de perda de controle, má coordenação, insônia, confusão, dor de cabeça, choro, fadiga, dores contínuas, cólicas, seios doloridos, compulsão por alimentos, inchaços, cabelos oleosos, acne, tontura, ânsia, euforia, isolamento, explosão de raiva, mastalgia ("seios doloridos"), desconforto abdominal, cefaleia, edema, dificuldade de concentração, cansaço, alteração do apetite e dor lombar (GAION; VIEIRA, 2010; DAVID et al., 2009; GAION; VIEIRA; SILVA, 2009; VIEIRA; GAION, 2009).

Sabe-se que os hormônios femininos, estrogênio e progesterona, têm efeitos potenciais sobre a capacidade de desempenhar um exercício por meio de inúmeros mecanismos, e as alterações hormonais podem interferir no desempenho atlético ao longo do CM. Estar consciente dessa influência sobre o desempenho físico é uma das maneiras de evitar sobrecarga desnecessária para a atleta, além de uma possível perda de performance durante as competições, respeitar a individualidade de cada mulher é primordial nesse contexto (DAVID et al., 2009).

Nesta pesquisa, quatro artigos (GAION; VIEIRA, 2010; DAVID et al., 2009; GAION; VIEIRA; SILVA, 2009; VIEIRA; GAION, 2009) relataram prejuízos na performance das atletas estudadas relacionada a SPM, com relato dos seguintes sintomas: ansiedade, tensão nervosa, irritabilidade, choro, insônia, depressão, mudanças de humor, cefaleia, fadiga, dores contínuas, cólicas, dores nos seios, inchaços, compulsão por alimentos.

Para que um programa seja eficaz e promova adaptações no organismo, faz-se necessário que haja a utilização de alguns princípios como: individualidade biológica, adaptação, sobrecarga, continuidade, especificidade e interdependência volume-intensidade são indispensáveis para a preparação física, uma vez que, bem assimilados, permitem que o treinador elabore seus próprios métodos com base nos métodos já existentes (DANTAS, 2003). Os fatores indispensáveis para a prescrição de um bom e seguro programa de treinamento são a frequência semanal, a duração e intensidade de cada período de treinamento (COSTILL; WILMORE, 2001).

A presente pesquisa foi composta somente de publicações na língua portuguesa para melhor delinear o problema em questão, que é a influência do CM na performance de atletas, entre pesquisas realizadas no Brasil, porém se tornou 
uma limitação do estudo, pois houve restrições nas possíveis palavras-chave que explicassem a temática de interesse, sendo necessária a utilização de sinônimos para fundamentar a pesquisa. Outra limitação da pesquisa foi que os artigos encontrados se utilizaram de um corte transversal para relatar os resultados, fato que com estudos do tipo longitudinal poderiam ser encontradas informações mais esclarecedoras e reais com relação às causas e consequências do CM na performance esportiva. Também, atualmente o uso de métodos contraceptivos é maior entre as mulheres para prevenção de doenças ou uma gravidez indesejada e, com isso os sinais e sintomas deste período no corpo feminino fica anulado, outro fato que dificultaria o relato de possíveis sintomas indesejados para não influenciar um treinamento desportivo. Outro ponto importante pode ser a relação interpessoal da atleta com o treinador, pelo fato de a atleta relatar possíveis desconfortos corporais no CM e o treinador excluí-la dos treinamentos, situação que poderia levar a atleta a omitir reais condições de saúde e, consequentemente comprometer a performance física e também os resultados numa pesquisa científica.

\section{Considerações Finais}

Assim, constatou-se que o CM interfere no sistema hormonal e em sintomas físicos e emocionais relatados pelas atletas, principalmente na fase pré-menstrual, podendo causar a SPM, e com impacto no desempenho físico. Sugere-se futuras pesquisas para que se verifique principalmente em qual das fases do CM existe um maior ganho de performance das diferentes capacidades físicas. Por fim, considerando que no esporte, tanto amador quanto de alto rendimento, qualquer fator passa a ser decisivo para o melhor desempenho, é importante que técnicos e profissionais que atuam com equipes femininas estejam atentos às questões relacionadas ao CM.

\section{Referências}

BISI, F. B. et al. Influência do ciclo menstrual na flexibilidade de atletas que utilizam contraceptivo oral. Rev. Bras. Cienc. Movimento, v. 17, n. 3, p. 18-24, 2009.

BORRESEN, J.; LAMBERT, M. I. The quantification of training load, the training response and the effect on performance. Am. J. Sports Med. v. 39, n. 9, p. 779-795, 2009 .

BORST, S. E. et al. Effects of resistance training on insulinlike growth factor-I and IGF binding proteins. Med. Sci. Sports Exerc. v. 33, n. 4, p. 648-653, 2001.

BRAUN, B.; HORTON, T. Endocrine regulation of exercise substrate utilization in women compared to men. Exercise and sport sciences reviews, v. 29, n. 4, p. 149-154, 2001.

BURINI, F. H. P.; OLIVEIRA, E. P. D.; BURINI, R. C. (Mal) adaptações metabólicas ao treinamento contínuo: concepções não consensuais de terminologia e diagnóstico. Rev. Bras. Med. Esporte, v. 16, n. 5, p. 388-392, 2010.

CARVALHO, J. C. de et al. A influência do ciclo menstrual no desempenho de atletas de futebol feminino. Rev. Eletrônica Saúde e Ciênc. v. 4, n. 1, p. 35-44, 2014.

CESAR, M. C.; PARDINI, D. P.; BARROS, T. L. Efeitos do exercício de longa duração no ciclo menstrual, densidade óssea e potência aeróbica de corredoras. Rev. Bras. Cienc. Movimento, v. 9, n. 2, p. 7-13, 2001.

CHAVES, C. P. G.; SIMÃO, R.; ARAÚJO, C. G. S. D. Ausência de variação da flexibilidade durante o ciclo menstrual em universitárias. Rev. Bras. Med. Esporte, v. 8, n. 6, p. 212-218, 2002.

COELHO, S. M. H.; SIMÕES, R. D.; LUNZ, W.

Desequilíbrio hormonal e disfunção menstrual em atletas de ginástica rítmica. Rev. Bras. Ciênc. Esporte, v. 37, n. 3, p. 222-229, 2015.

COSTILL, D. L.; WILMORE, J. H. Fisiologia do esporte e do exercício. São Paulo: Manole, 2001.

DANTAS, E. H. M. A prática da preparação física. 5. ed. Rio de Janeiro: Shape, 2003.

DAVID, A. M. et al. Incidência da síndrome pré-menstrual na prática de esportes. Rev. Bras. Med. Esporte, v. 15, n. 5, p. 330-333, 2009.

DIAS, I. B. F.; SIMÃO, R.; DA SILVA NOVAES, J. Efeito das diferentes fases do ciclo menstrual em um teste de 10RM. Fit. Perf. J. v. 4, n. 5, p. 288-292, 2005.

DIFERENÇAS entre homens e mulheres no esporte: as diferenças entre os sexos no esporte são muitas, principalmente nas atividades em que determinadas capacidades são decisivas, como resistência. Disponível em: <http://www.educacaofisica.com.br/saude-bem-estar/ diferencas-entre-homens-e-mulheres-no-esporte/>. Acesso em: 07 out. 2015.

BARROS, Turibio. Diferenças da genética entre homens e mulheres influencia nas atividades: em esportes como maratonas aquáticas, mulheres poderiam competir de igual para igual com os homens. Gordura corporal feminina ajuda na flutuabilidade. Disponível em: < http://globoesporte. globo.com/eu-atleta/saude/noticia/2013/07/diferencada-genetica-entre-homens-e-mulheres-influenciam-nasatividades.html>. Acesso em: 23 out. 2017.

FERNANDES, J. S.; FORTUNATO, J. M. S.; CORREIAPINTO, J. Fisiologia do sistema reprodutor feminino. SOF-Fisiologia. Universidade do Minho. 45 p.

FLECK, Steven J.; KRAEMER, William. Designing resistance training programs, 4E. Human Kinetics, 2014.

FREITAS, V. H. D.; MILOSKI, B.; BARA FILHO, M. G. Quantificação da carga de treinamento através do método de percepção subjetiva do esforço da sessão e desempenho no futsal. Rev. Bras. Cineantropom. Desempenho Hum. v. 14, n. 1, p. 73-82, 2011. 
GAION, P.; VIEIRA, L. F.; SILVA, C. M. L. Síndrome prémenstrual e percepção de impacto no desempenho esportivo de atletas brasileiras de futsal. Rev. Bras. Cineantropom.

Desempenho Hum. v. 11, n. 1, p. 73-80, 2009.

GAION, P. A.; VIEIRA, L. F. Prevalência de síndrome prémenstrual em atletas. Rev. Bras. Med. Esporte, v. 16, n. 1, p. 24-28, 2010.

GLASS, S. C.; STANTON D. R. Self-selected resistance training intensity in novice weightlifters. J. Strength Cond. Res. v. 18, n. 2, p. 324-327, 2004.

GOELLNER, S. V. Mulher e esporte no Brasil: entre incentivos e interdições elas fazem história. Pensar a prática, v. 8, n. 1, p. 85-100, 2006.

GUYTON, A. C.; HALL, J. E.; GUYTON, A. C. Tratado de fisiologia médica. São Paulo: Elsevier Brasil, 2006.

JONGE, X. A. J. de Effects of the menstrual cycle on exercise performance. Sports Medicine, v. 33, n. 11, p. 833-851, 2003.

JUNQUEIRA, Nívia de Oliveira. Influência da corrida no ciclo menstrual. Disponível em: $<$ https:// www.portaleducacao.com.br/conteudo/artigos/ educacao-fisica/influencia-da-corrida-no-ciclomenstrual/16992\#ixzz3mlZakLAe>. Acesso em: 26 jun. 2015.

LOUREIRO, S. et al. Efeito das diferentes fases do ciclo menstrual no desempenho da força muscular em 10RM.

Rev. Bras. Med. Esporte, v. 17, n. 1, p. 22-25, 2011.

MANTOANELLI, G.; VITALLE, M. S. D. S.; AMANCIO, O. M. S. Amenorreia e osteoporose em adolescentes atletas. Rev. Nutr. v. 15, n. 3, p. 319-340, 2002.

MELEGARIO, S. M. et al. A influência do ciclo menstrual na flexibilidade em praticantes de ginástica de academia.

Rev. Bras. Med. Esporte, v. 12, n. 3, p. 125-128, 2006.

NAKAMURA, F. Y.; MOREIRA, A.; AOKI, M. S. Monitoramento da carga de treinamento: a percepção subjetiva do esforço da sessão é um método confiável? Rev. da Educação Física/UEM, v. 21, n. 1, p. 1-11, 2010.

NUNES, J. A. et al. Monitoramento da carga interna no basquetebol. Rev. Bras. Cineantropom. Desempenho Hum. v. 13, n. 1, p. 67-72, 2011.

PARDINI, D. P. Alterações hormonais da mulher atleta. Arq. Bras. Endocrinol. Metab. 45, n. 4, p. 343-351, 2001.

SALVADOR, E. P. et al. Comparação entre o desempenho motor de hommes e mulheres em séries múltiplas de exercícios com pesos. Rev. Bras. de Med. Esporte, v. 11, n. 5 , p. 257-261, 2005.

SANBORN, C. F.; MARTIN, B. J.; WAGNER, W. W. Is athletic amenorrhea specific to runners? Am. J. Obstet. Gynecol. v. 143, n. 8, p. 859-861, 1982.

SCHTSCHERBYNA, A. et al. A idade do início do treinamento, e não a composição corporal, está associada com disfunções menstruais em nadadoras adolescentes competitivas. Rev. Bras. Med. Esporte, v. 18, n. 3, p. 161 $163,2012$.

SELYE, H. The effect of adaptation to various damaging agents on the female sex organs in the rat. Endocrinology, v. 25 , n. 4 , p. $615-624,1939$.

TEIXEIRA, A. L. D. S. et al. Influência das diferentes fases do ciclo menstrual na flexibilidade de mulheres jovens. Rev Bras Med Esporte, v. 18, n. 6, p. 361-364, 2012.

TEIXEIRA, A. L. D. S.; OLIVEIRA, É. C. M.; DIAS, M. R. C. Relação entre o nível de atividade física e a incidência da síndrome pré-menstrual. Rev. Bras. Ginecol. Obstet. v. 35 , n. 5 , p. $210-214,2013$.

VIEIRA, L. F.; GAION, P. A. Impacto da síndrome prémenstrual no estado de humor de atletas. J. Bras. Psiquiatr. v. 58, n. 2, p. 101-106, 2009.

WOJTYS, E. M. et al. Association between the menstrual cycle and anterior cruciate ligament injuries in female athletes. The American Journal of Sports Medicine, v. 26, n. 5, p. 614-619, 1998. 